\title{
Erratum: Calculations of spin response functions in rings with Siberian Snakes and spin rotators [Phys. Rev. ST Accel. Beams 12, 024001 (2009)]
}

\author{
Yu. M. Shatunov and S. R. Mane \\ (Received 9 March 2009; published 18 March 2009) \\ PACS numbers: 29.27.Hj, 29.20.D-, 02.60.Lj, 99.10.Cd
}

DOI: 10.1103/PhysRevSTAB.12.039902

The values of $\arg \left(F_{3}\right)$ in the plots in Figs. 5 and 7 of our paper (beam energies of 205 and $250 \mathrm{GeV}$, respectively) were inadvertently divided by a factor of $2 \pi$. We display the corrected graphs below. The graph for $\arg \left(F_{3}\right)$ for a beam energy of $100 \mathrm{GeV}$ (Fig. 3 of our paper) is correct, as are all the graphs for the amplitude $\left|F_{3}\right|$ (Figs. 1, 2, 4, and 6). As a result of this correction, the statement in our paper must be amended: "We also see that, at both 205 and $250 \mathrm{GeV}$, the phase of $F_{3}$ changes relatively little across the drift space, for all three scenarios. This is significantly different from the behavior of the phase of $F_{3}$ at $100 \mathrm{GeV}$." In fact the phase of $F_{3}$ can change by $90^{\circ}$ or more, at all the beam energies that we studied. Moreover, this fact emphasizes the necessity to pay more attention to the choice of the flipper parameters. Our overall conclusion, that the value of $F_{3}$ (both amplitude and phase) depends sensitively on the beam energy and spin rotator settings, remains valid.
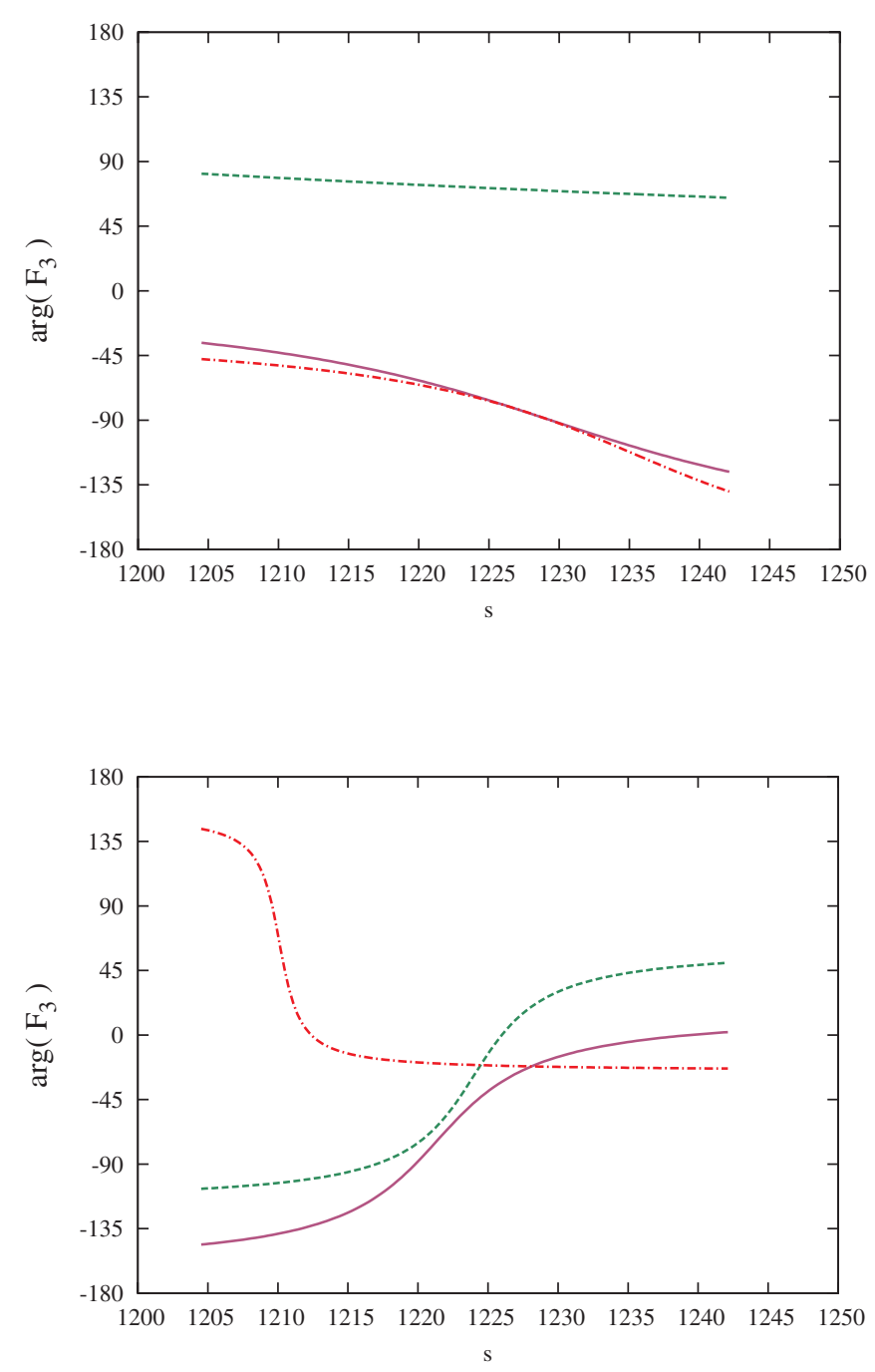

FIG. 5. (Color) Graph of $\arg \left(F_{3}\right)$ at $205 \mathrm{GeV}$ in the drift space L232 of RHIC for three scenarios: (a) solid (purple) —no rotators, (b) dashed (green)-longitudinal polarization at both STAR and PHENIX, (c) dot-dashed (red)—no rotators at STAR, radial polarization at PHENIX. The horizontal axis shows the arc length in meters, measured from the 6 o'clock point.
FIG. 7. (Color) Graph of $\arg \left(F_{3}\right)$ at $250 \mathrm{GeV}$ in the drift space L232 of RHIC for three scenarios: (a) solid (purple)—no rotators, (b) dashed (green)-longitudinal polarization at both STAR and PHENIX, (c) dot-dashed (red)—no rotators at STAR, radial polarization at PHENIX. The horizontal axis shows the arclength in meters, measured from the 6 o'clock point. 